\title{
Photorefractive damage resistance threshold in stoichiometric $\mathrm{LiNbO}_{3}: \mathrm{Zr}$ crystals
}

\author{
László Kovács, ${ }^{1, *}$ Zsuzsanna Szaller, ${ }^{1}$ Krisztián Lengyel, ${ }^{1}$ Ágnes Péter, ${ }^{1}$ Ivett Hajdara, ${ }^{1}$ \\ Gábor Mandula, ${ }^{1}$ László Pálfalvi, ${ }^{2}$ and János Hebling ${ }^{2,3}$ \\ ${ }^{1}$ Wigner Research Centre for Physics, Hungarian Academy of Sciences, H-1121 Budapest, Konkoly-Thege M. út 29-33, Hungary \\ ${ }^{2}$ Institute of Physics, University of Pécs, H-7624 Pécs, Ifjúság útja 6, Hungary \\ ${ }^{3}$ MTA-PTE High-Field Terahertz Research Group, H-7624 Pécs, Ifjúság útja 6, Hungary \\ "corresponding author: kovacs.laszlo@wigner.mta.hu
}

Received May 17, 2013; revised June 26, 2013; accepted June 30, 2013;

posted July 3, 2013 (Doc. ID 189594); published July 30, 2013

\begin{abstract}
Several optical methods including ultraviolet absorption, infrared absorption of the hydroxyl ions, Raman spectroscopy, and the Z-scan method have been used to determine the damage resistance threshold in 0-0.72 mol. \% Zr-containing, flux-grown, nearly stoichiometric $\mathrm{LiNbO}_{3}$ single crystals. All spectroscopical methods used indicate that samples containing at least $\approx 0.085 \mathrm{~mol} . \% \mathrm{Zr}$ in the crystal are above the threshold while Z-scan data locate the photorefractive damage threshold between 0.085 and 0.314 mol. \% Zr. (C) 2013 Optical Society of America

OCIS codes: (160.5320) Photorefractive materials; (190.4400) Nonlinear optics, materials; (300.6340) Spectroscopy, infrared; (300.6450) Spectroscopy, Raman; (300.6540) Spectroscopy, ultraviolet.

http://dx.doi.org/10.1364/OL.38.002861
\end{abstract}

The suppression of optical damage related to photorefraction is one of the most important objectives for ferroelectric $\mathrm{LiNbO}_{3}$ (LN) devices. The optical damage resistance of LN crystals can be improved by doping with certain divalent, trivalent, or tetravalent cations (e.g., $\mathrm{Mg}^{2+}, \mathrm{Zn}^{2+}, \mathrm{In}^{3+}, \mathrm{Sc}^{3+}, \mathrm{Hf}^{4+}$ ) above a critical amount [1]. Increased damage resistance in the visible optical range has been reported recently in tetravalent Zr- [2,3] and Sn-doped [4] LN crystals as well. Ferroelectric domain engineering with auxiliary UV light is of growing interest in damage-resistant lithium niobate [5]. High resistance against UV photorefraction has been reported in Zr-doped LN [6]. However, for the Hf and Sn dopant enhanced UV photorefractive properties, especially at the band edge, have been observed [7,8]; a summary was recently given by Kong et al. [9].

For LN grown from congruent melt the threshold concentration of the optical damage resistant (ODR) ions is roughly 5,4 , and $2 \mathrm{~mol}$. \% for di-, tri-, and tetravalent ions, respectively. For example, the threshold concentration for Zr-doped congruent LN crystals was found to be at about 2 mol. \% [3, 10,11]. Another factor beside the ODR ions and their valency strongly effecting the threshold concentration is stoichiometry of the crystal. The incorporation of ODR ions reduces the amount of antisite $\mathrm{Nb}_{\mathrm{Li}}$ in the lattice. In nearly stoichiometric $\mathrm{LiNbO}_{3}(\mathrm{sLN})$ crystals where the $\mathrm{Nb}_{\mathrm{Li}}$ concentration is almost zero, the threshold value of the dopant concentration can be lower than 0.2 mol. \% as was observed for Mg-doped sLN [12] used for instance for terahertz pulse generation [13]. Zr-doped nearly stoichiometric LN crystals prepared by the vapor transport equilibration method were found to have high resistance against optical damage when doped above $0.5 \mathrm{~mol}$. \% $\mathrm{Zr}$ [14]. The basic advantage of choosing tetravalent ODR dopants comes from their lower damage threshold concentrations; lower built-in dopant content facilitates the growth of more homogeneous crystals, resulting in high-quality samples for device applications.

The aim of the present work is to characterize the damage resistance properties of tetravalent $\mathrm{Zr}$-doped sLN crystals by ultraviolet (UV), infrared (IR), and Raman spectroscopies as well as by the Z-scan method. These methods proved earlier very efficient to determine the threshold concentration of the dopants.

A series of stoichiometric LN crystals doped with $\mathrm{Zr}$ in the $0-0.45 \mathrm{~mol}$. \% concentration range were grown by the high-temperature top-seeded solution growth method using $\mathrm{K}_{2} \mathrm{O}$ flux [12]. The $\mathrm{Zr}$ concentration in the crystal was determined by inductively coupled plasma mass spectroscopy (ICP-MS). As shown in Fig. 1, the amount of incorporated $\mathrm{Zr}$, at least in the range $0-0.715 \mathrm{~mol}$. \% realized in our samples, monotonously increases with increasing concentration of $\mathrm{Zr}$ in the starting flux. The relatively high distribution coefficient of $\mathrm{Zr}$ as compared to that observed in the melt-grown congruent crystals [3] may be related to the different growing technique, similarly to the case of $\mathrm{Mg}[\underline{12}]$.

For optical spectroscopical measurements 1-2 mm thick $z$-and $y$-cut samples have been prepared. The UV-visible absorption spectra of $z$-cut sLN:Zr crystals were measured by a Jasco V-550 spectrometer at room temperature. The spectra of the stretching vibrational bands of the hydroxyl ions $\left(\mathrm{OH}^{-}\right)$in $\mathrm{sLN}: \mathrm{Zr}$ were

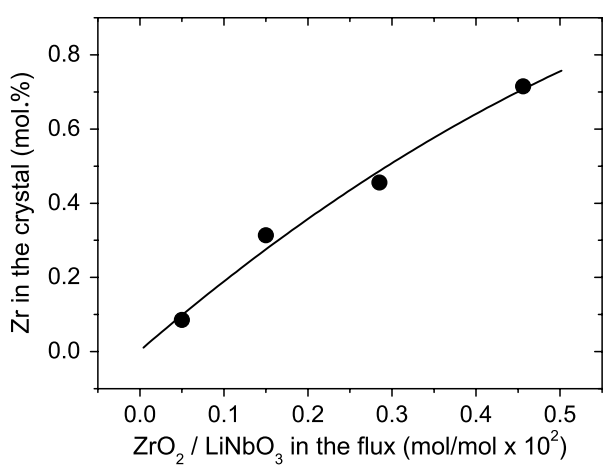

Fig. 1. $\mathrm{Zr}$ concentration in stoichiometric $\mathrm{LiNbO}_{3}$ crystal as a function of the concentration in the flux. Errors are smaller than or comparable to symbol sizes. 
recorded by a Bruker IFS 66v/S FTIR spectrometer. The Raman spectra of sLN:Zr crystals were measured in backscattering geometry by a Renishaw 1000 B microRaman spectrometer using a $785 \mathrm{~nm}$ laser source. The light-induced refractive index change was investigated by the Z-scan method using a standard single-beam setup with an all-lines visible argon ion laser with dominant wavelengths of 514 and $488 \mathrm{~nm}$ and intensities up to $300 \mathrm{~kW} / \mathrm{cm}^{2}$.

The UV absorption edge of $\mathrm{LiNbO}_{3}$ is very sensitive to the $\mathrm{Li} / \mathrm{Nb}$ ratio of the crystal, that is, the concentration of $\mathrm{Nb}_{\mathrm{Li}}$ antisite niobium, and to the incorporated ODR ions $[15,16]$. The UV edge is usually defined as the wavelength where the absorption coefficient value reached $20 \mathrm{~cm}^{-1}$. The observed UV edge values monotonously increased between 304.2 and $305.4 \mathrm{~nm}$ with increasing $\mathrm{Zr}$ concentration (Fig. 2) in good agreement with the data of Liu et al. [14]. According to the simple, widely accepted model the ODR dopants push out the antisite $\mathrm{Nb}$ ions from the Li sites up to the threshold concentration where no $\mathrm{Nb}_{\mathrm{Li}}$ ions are left in the crystal. Thus the $\mathrm{Li} / \mathrm{Nb}$ ratio increases up to the threshold, which results in a shift of the UV edge toward shorter wavelengths [15]. The incorporation of ODR ions by itself causes a redshift of the UV edge which can clearly be detected above the threshold as described in detail for sLN:Mg [16]. Consequently the observed redshift of the UV edge in sLN:Zr is characteristic for crystals containing $\mathrm{Zr}$ ions above the threshold concentration. While the shift of the UV edge for divalent $\mathrm{Mg}$ is about $1 \mathrm{~nm} / \mathrm{mol}$.\%, it is about twice this amount for tetravalent Zr-doped sLN.

Another very sensitive method to study the incorporation of ODR ions in $\mathrm{LiNbO}_{3}$ is the IR absorption spectroscopy of hydroxyl ions always present in air-grown
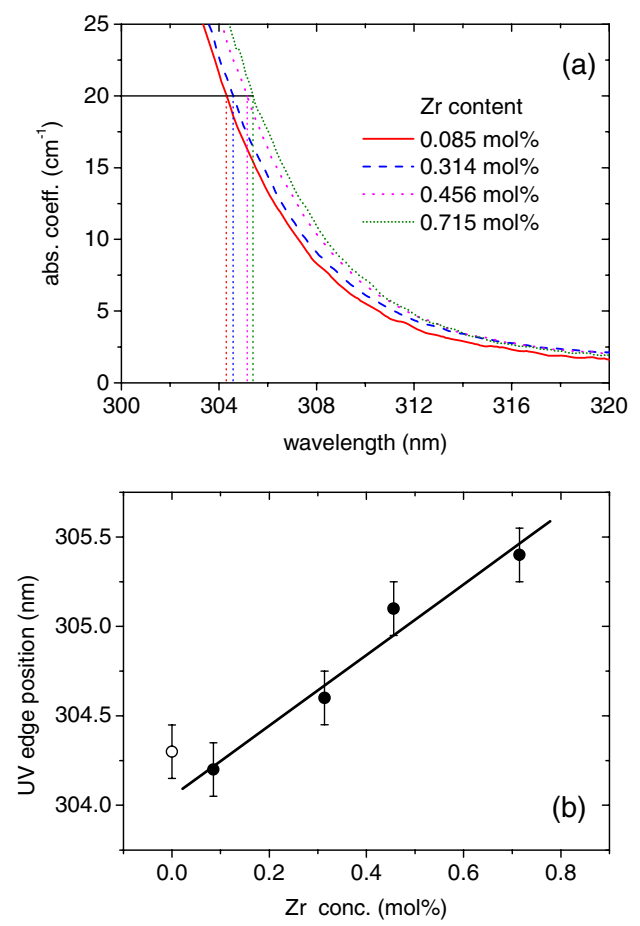

Fig. 2. UV absorption edge as a function of $\mathrm{Zr}$ concentration in stoichiometric $\mathrm{LiNbO}_{3}$ crystals. The open circle corresponds to an undoped sLN crystal with $\mathrm{Li}_{2} \mathrm{O}$ content equal to $49.96 \mathrm{~mol}$. \%. crystals. In crystals above the photorefractive threshold a new absorption band related to $\mathrm{OH}^{-}$ions appears due to their stretching vibration in a $\mathrm{M}_{\mathrm{Nb}}^{n+}-\mathrm{OH}^{-}$complex, where $\mathrm{M}_{\mathrm{Nb}}^{n+}$ is a di-, tri-, or tetravalent ODR ion occupying an $\mathrm{Nb}$ site in the lattice [1]. For congruent $\mathrm{LiNbO}_{3}$ this band is not always clearly seen due to the overlapping broad absorption band characteristic for the pure crystal. However, the pure stoichiometric crystal contains a single narrow band at about $3466 \mathrm{~cm}^{-1}$ which allows the identification of nearby new bands. In Zr-doped sLN, beside the $3466 \mathrm{~cm}^{-1}$ band present also in this crystal together with a weak shoulder at about $3480 \mathrm{~cm}^{-1}$ characteristic for some kind of residual disorder in the crystal, the appearance and the continuous growth of an $\mathrm{OH}^{-}$ vibrational band at $3475 \mathrm{~cm}^{-1}$ was observed (see Fig. 3). The relative intensity of the $\mathrm{Zr}_{\mathrm{Nb}}^{4+}-\mathrm{OH}^{-}$band increased with increasing $\mathrm{Zr}$ content, confirming its assignation. Kong et al.. reported the appearance of the $\mathrm{Zr}-\mathrm{OH}$ band at about $3487 \mathrm{~cm}^{-1}$ in congruent $\mathrm{Zr}$-doped crystal, but no spectrum was presented in their paper [3]. Fan et al. studied $\mathrm{Zr}$ and $\mathrm{Fe}$ codoped $\mathrm{LiNbO}_{3}$ with various $\mathrm{Li} / \mathrm{Nb}$ ratios, and in a crystal grown from a melt with $\mathrm{Li} / \mathrm{Nb}=$ 1.2 they found a vibrational band at $3475 \mathrm{~cm}^{-1}$, which they attributed to $\mathrm{Zr}_{\mathrm{Nb}}-\mathrm{OH}$ complexes [17], in agreement with our observation.

The Raman spectra of sLN:Zr crystals were measured in $y(z z) y$ and $y(x z) y$ backscattering geometries between 70 and $1000 \mathrm{~cm}^{-1}$ [Fig. 4(a)]. The $\mathrm{A}_{1}$ (TO) and $\mathrm{E}(\mathrm{TO})$ modes observed are sensitive to the $\mathrm{Li} / \mathrm{Nb}$ ratio and
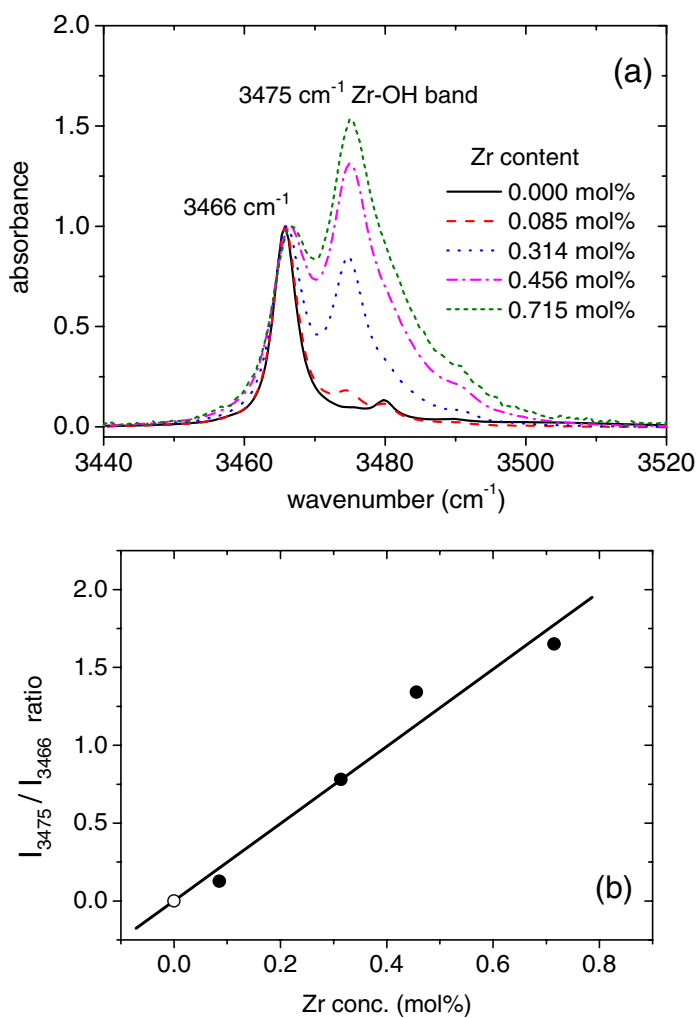

Fig. 3. (a) Stretching vibrational bands of hydroxyl ions in Zr-doped stoichiometric $\mathrm{LiNbO}_{3}$ and (b) intensity ratio of the two bands, as a function of $\mathrm{Zr}$ concentration. The spectra were normalized to the $\mathrm{OH}$ band at $3466 \mathrm{~cm}^{-1}$. The open circle in Fig. 3(b) corresponds to the undoped sLN crystal where no $\mathrm{Zr}_{\mathrm{Nb}}^{4+}-\overline{\mathrm{OH}^{-}}$band at $3475 \mathrm{~cm}^{-1}$ can be observed. 
(a)
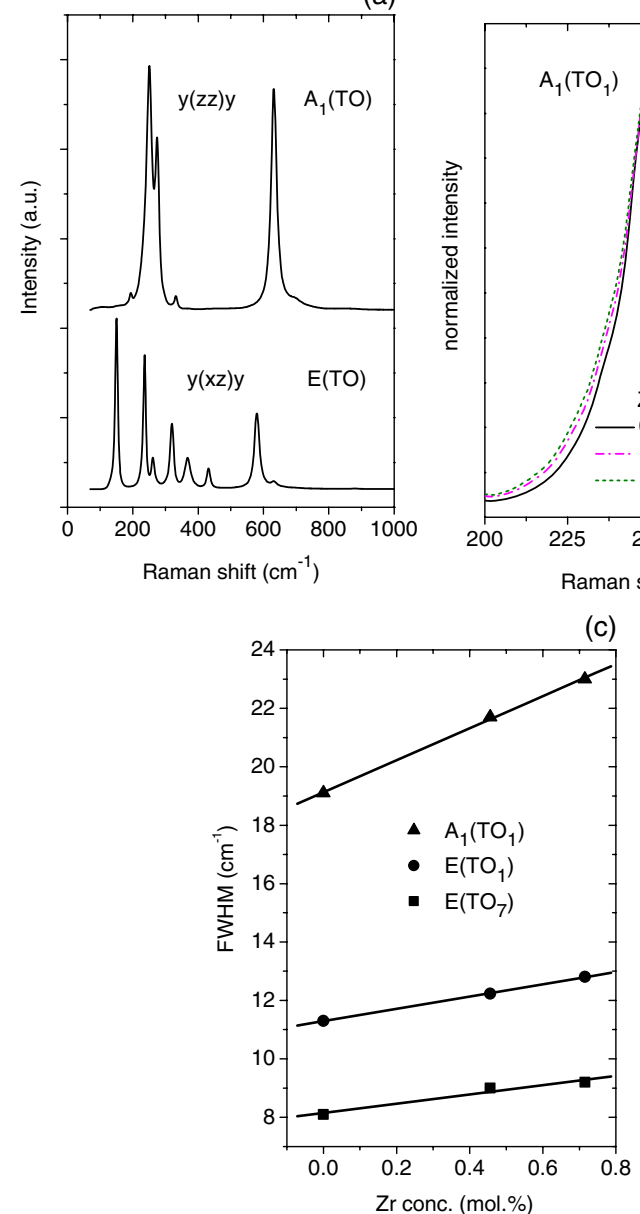

Fig. 4. (a) Raman spectra of $\mathrm{Zr}$-doped stoichiometric $\mathrm{LiNbO}_{3}$ crystals. $\mathrm{A}_{1}(\mathrm{TO})$ and $\mathrm{E}(\mathrm{TO})$ modes measured in $y(z z) y$ and $y(x z) y$ geometry, respectively; (b) broadening of the $\mathrm{A}_{1}\left(\mathrm{TO}_{1}\right)$ and $\mathrm{A}_{1}\left(\mathrm{TO}_{2}\right)$ modes, and (c) widths of several Raman lines as a function of $\mathrm{Zr}$ concentration in the crystal.

the concentration of the ODR dopants. As an example, Fig. 4(b) shows the damping of the $\mathrm{A}_{1}\left(\mathrm{TO}_{1}\right)$ and $\mathrm{A}_{1}\left(\mathrm{TO}_{2}\right)$ modes between 200 and $300 \mathrm{~cm}^{-1}$ as a function of $\mathrm{Zr}$ concentration. The higher the amount of $\mathrm{Zr}$ in the crystal, the broader the Raman lines. Similar broadening was also observed, for example, for the $\mathrm{E}\left(\mathrm{TO}_{1}\right)$ and $\left.\mathrm{E}\left(\mathrm{TO}_{7}\right)\right)$ modes [Fig. 4(c)]. These results are in good agreement with those measured on Mg-doped SLN crystals [18]. The damping of Raman modes linearly increases with the $\mathrm{Zr}$ content, which corresponds to over-threshold behavior as described in [18].

The light-induced refractive index change was investigated by the Z-scan method. It was shown by Pálfalvi et al. [19] that Z-scan measurements can be used to distinguish crystals with Mg doping levels below and above the photorefractive threshold. Below the threshold the photorefractive effect was found to be dominant with negative refractive index change resulting in Z-scan curves with a peak-valley sequence. On the contrary, above the threshold the Z-scan curves with a valley-peak sequence showed a positive refractive index change caused by thermal effects. As clearly seen in Fig. $\underline{5}$, only the $0.085 \mathrm{~mol}$. \% Zr-containing sample shows photorefractive damage, while those having higher $\mathrm{Zr}$ content

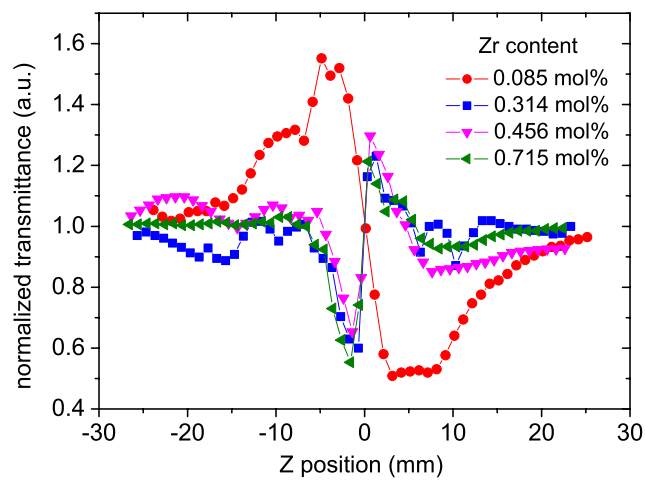

Fig. 5. Z-scan curves of Zr-doped stoichiometric $\mathrm{LiNbO}_{3}$ crystals measured with $310 \mathrm{~kW} / \mathrm{cm}^{2}$ intensity.

reveal Z-scan curves with opposite sign and are well above the threshold.

All methods (Z-scan, UV, IR, and Raman) used to characterize the sLN:Zr crystals indicate that almost all investigated samples are at or above the photorefractive damage threshold. Although the threshold is assumed to appear at the doping concentration where all antisite $\mathrm{Nb}$ ions disappear from the crystal, the transition between the below- and above-threshold behavior may not be sharply defined. The Z-scan measurement showed clearly that the crystal containing $0.085 \mathrm{~mol}$. \% $\mathrm{Zr}$ is below the photorefractive threshold, while the results obtained from other methods indicate that the threshold is below or at this concentration. A similar observation was presented by Nava et al. for congruent Zr-doped $\mathrm{LiNbO}_{3}$ crystals [11], showing that the photorefractivity is reduced at somewhat higher $\mathrm{Zr}$ concentration than the threshold determined from refractive index measurements. Nevertheless the earlier expectations are confirmed: the closer the crystal to the stoichiometric composition, the lower the attainable threshold. The extremely low damage threshold found for tetravalent $\mathrm{Zr}$ in stoichiometric lithium niobate is expected to facilitate the growth of high-quality homogeneous crystals for device applications.

The authors are grateful to Krisztina György for the digestion of the samples and also to Kornél Fél and Éva Széles for ICP-MS analyses made at the Institute for Isotope Research, Centre for Energy Research. Fruitful discussions with Dr. Gábor Corradi are also acknowledged. The work has been supported by the Hungarian Research Fund (OTKA K-83390) and SROP-4.2.2.A-11/ 1Scientific/KONV-2012-0065.

\section{References}

1. T. Volk and M. Wöhlecke, Lithium Niobate, Vol. 115 of Springer Series in Material Science (Springer, 2008).

2. L. Sun, F. Guo, Q. Lv, H. Yu, W. Cai, Y. Xu, and L. Zhao, Cryst. Res. Technol. 42, 1117 (2007).

3. Y. Kong, S. Liu, Y. Zhao, H. Liu, S. Chen, and J. Xu, Appl. Phys. Lett. 91, 081908 (2007).

4. L. Wang, S. Liu, Y. Kong, S. Chen, Z. Huang, L. Wu, R. Rupp, and J. Xu, Opt. Lett. 35, 883 (2010).

5. H. Steigerwald, F. Luedtke, and K. Buse, Appl. Phys. Lett. 94, 032906 (2009).

6. F. Liu, Y. Kong, W. Li, H. Liu, S. Liu, S. Chen, X. Zhang, R. Rupp, and J. Xu, Opt. Lett. 35, 10 (2010). 
7. F. Xin, G. Zhang, F. Bo, H. Sun, Y. Kong, J. Xu, T. Volk, and N. M. Rubinina, J. Appl. Phys. 107, 033113 (2010).

8. F. Xin, G. Zhang, X. Ge, S. Liu, L. Xuan, Y. Kong, and J. Xu, Opt. Lett. 36, 3163 (2011).

9. Y. Kong, S. Liu, and J. Xu, Materials 5, 1954 (2012).

10. N. Argiolas, M. Bazzan, M. V. Ciampolillo, P. Pozzobon, C. Sada, L. Soaner, A. M. Zaltron, L. Bacci, P. Minzioni, G. Nava, J. Parravicini, W. Yan, I. Cristiani, and V. Degiorgio, J. Appl. Phys. 108, 093508 (2010).

11. G. Nava, P. Minzioni, W. Yan, J. Parravicini, D. Grando, E. Musso, I. Cristiani, N. Argiolas, M. Bazzan, M. V. Ciampolillo, A. Zaltron, C. Sada, and V. Degiorgio, Opt. Mater. Express 1, 270 (2011).

12. Á. Péter, K. Polgár, L. Kovács, and K. Lengyel, J. Cryst. Growth 284, 149 (2005).
13. L. Pálfalvi, J. Hebling, J. Kuhl, Á. Péter, and K. Polgár, J. Appl. Phys. 97, 123505 (2005).

14. H. Liu, Q. Liang, M. Zhu, W. Li, S. Liu, L. Zhang, S. Chen, Y. Kong, and J. Xu, Opt. Express 19, 1743 (2011).

15. L. Kovács, G. Ruschhaupt, K. Polgár, G. Corradi, and M. Wöhlecke, Appl. Phys. Lett. 70, 2801 (1997).

16. K. Lengyel, Á. Péter, K. Polgár, L. Kovács, and G. Corradi, Phys. Status Solidi C 2, 171 (2005).

17. Y. Fan, C. Xu, S. Xia, C. Guan, L. Cao, Q. He, and G. Jin, J. Cryst. Growth 312, 1875 (2010).

18. K. Lengyel, L. Kovács, Á. Péter, K. Polgár, and G. Corradi, Appl. Phys. B 87, 317 (2007).

19. L. Pálfalvi, J. Hebling, G. Almási, Á. Péter, K. Polgár, K. Lengyel, and R. Szipócs, J. Appl. Phys. 95, 902 (2004). 